\title{
MHD stagnation-point flow and heat transfer towards stretching sheet with induced magnetic field
}

\begin{abstract}
The problem of the steady magnetohydrodynamic (MHD) stagnation-point flow of an incompressible viscous fluid over a stretching sheet is studied. The effect of an induced magnetic field is taken into account. The nonlinear partial differential equations are transformed into ordinary differential equations via the similarity transformation. The transformed boundary layer equations are solved numerically using the shooting method. Numerical results are obtained for various magnetic parameters and Prandtl numbers. The effects of the induced magnetic field on the skin friction coefficient, the local Nusselt number, the velocity, and the temperature profiles are presented graphically and discussed in detail.
\end{abstract}

Keyword: Boundary layer; Heat transfer; Induced magnetic field; Numerical solution; Magnetohydrodynamic (MHD) flow; Stretching sheet 